\title{
Research on Key Software Design Technologies of Target Seeker Performance Test System
}

\author{
Guangling Dong ${ }^{1, *}$, Cailing Wang ${ }^{2}$, Jing Wang ${ }^{1}$ and Chi $\mathrm{He}^{1}$ \\ ${ }^{1}$ Baicheng Ordnance Test Center of China, 108 Mail Box, Baicheng, Jilin, China \\ ${ }^{2}$ Library of Headquarters, 63680 Troops of PLA, Jiangyin, Jiangsu, China \\ ${ }^{*}$ Corresponding author
}

\begin{abstract}
Target seeker is a key component of missile, whose tracking performance in real guidance phases can be guaranteed by performance testing in its design stage. Generally, multi-sets of equipment are used in conventional testing method, which takes on some disadvantages of sophisticated operation and low levels of automation. To solve this problem, we developed an automatic test system of target seeker based on virtual instrument technology, which combines hardware units as PXI measuring and control platform, turntable system, target simulator, etc., together, and utilizes software design to achieve synchro dyne reception of serial port data, information acquisition \& display of video image and integrated control of turntable. Applications indicate that the system can accomplish automatic testing tasks, effectively reduce test period, and greatly improve automation and intelligent level in performance testing of target seeker.
\end{abstract}

Keywords-target seeker; performance testing; virtual instrument; PXI bus; multithreading

\section{INTRODUCTION}

As a vital component of missile, target seeker is mainly composed of coordinator and electronic cabin. It can accomplish autonomous searching, identifying and tracking of target in its operational process, and plays an important role in missile guidance and control. To guarantee the operational performance of missile, target seeker must be tested and adjusted before installation.

By now, performance-testing systems to infrared seeker have made great progress. In literatures [1-3], some vibration performance test systems of target seeker coordinator based on virtual instrument have been introduced. Zheng [4] put forward a target seeker measurement and control system using PXI bus. Chen [5] designed a kind of gyro rotor dynamic balance system of coordinator based on virtual instrument. Xin [6] suggested a design method for automatic test system of target seeker, and introduced its key technologies. Above-mentioned systems made full considerations of computer and digital bus technology in modern target seeker, which greatly improve automation level of testing. However, little attention has been paid to combined control of dynamic test conditions in operational process, especially in operations as measurement and control of platform equipment, parameters calculation, file $\mathrm{I} / \mathrm{O}$ and storage, extensive manual operations are still required.

Focusing on system automatization and intellectualization, we design a target seeker performance test system (TSPTS) based on PXI bus hardware platform and virtual instrument technology in this paper, which can be used in both comprehensive testing for overall performance of infrared seeker, and automatic calibration for coordinator parameters of rotary transformer, gyro and potentiometer zero position. Testing and calibration reports of target seeker can be generated automatically according to acquired data from qualification testing items.

\section{SYSTEM OVERVIEW}

\section{A. System Composition}

Figure I shows the composition of TSPTS, which uses proper adapters to connect PXI bus measurement and control platform and subsystems as turntable, target source, power supply module, air source module, etc., to realize various functions. Output and status information of target seeker, electronic building brick, turntable, target source, air source, etc. can be acquired and processed by the system. Acquisition, analysis and display processing function could automatically collect data of current, voltage, cooling flow rate, motion parameter, image, etc., which could also modify the display and analysis on each channel conveniently by adjusting the parameter display module. Performance testing and calibration function could display the acquired data, test results and waveform, which could also delete and backup data files. Platform control function could control attitude motion of main turntable, yawing rotation of target table, start and stop of air source, working mode switch of target seeker, etc. 


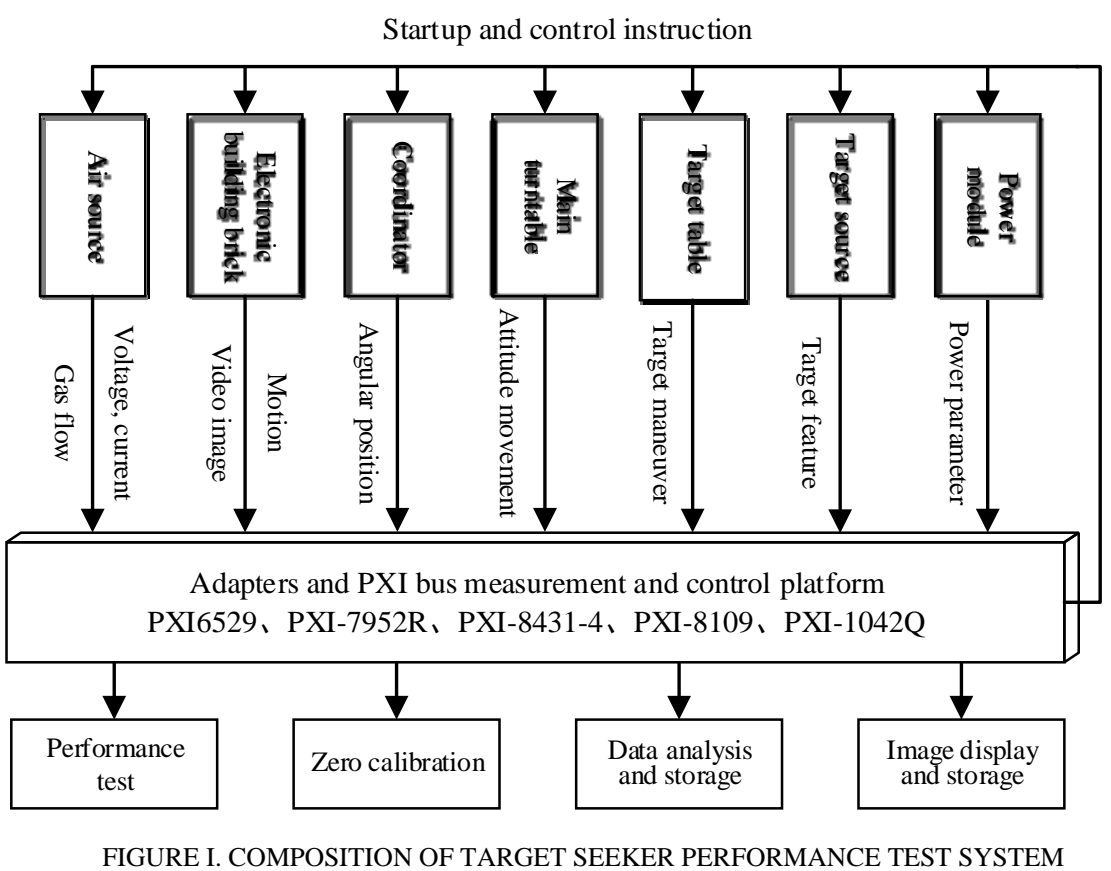

Target seeker adapter realizes exchange and preprocessing of signals between test system and the measured object. Measured signals are assembled here and then allocated to different modules, which serves as a link between target seeker and instrument platform. The adapter circuits include different function modules as digital signals driver, analog signal conditioning, isolation and amplification of pulsing signal, power supply changeover, failure predication, etc.

PXI bus technology of NI corporation is adopted in the instrument platform to constitute a solid automatic measurement platform based on PC, which combines electric features of PCI as well as sturdiness, modular and Eurocard mechanical capsulation of Compact PCI. Besides, PXI based instrument platform upgrade special sync bus and software feature to fit for the requirements of high performance and low-cost runtime platform. Instrument platform is mainly composed of multichannel data acquisition card PXI-6259, multiport serial card PXI-8431/4, and PXI-7952R FlexRIO FPGA module.
PXI-6259 is connected to coordinator, power supply module and air source to realize their signal acquisition and control. PXI-8431/4 serial card is connected to coordinator, turntable and aperture diaphragm controller of target source black body to realize relevant functions of control and information acquisition. PXI-7952R FlexRIO FPGA module completes the serial-parallel conversion of LVDS data acquired from coordinator.

\section{B. Software Design of System}

Virtual instrument technology [5, 7] is a kind of customer designed computer instrument system having virtual panels, which takes universal computer as kernel hardware platform, and realizes its test functions through software. Virtual instrument technology and LabWindows/CVI development platform [8] are used in design of test system, whose software design framework is shown in Figure II.

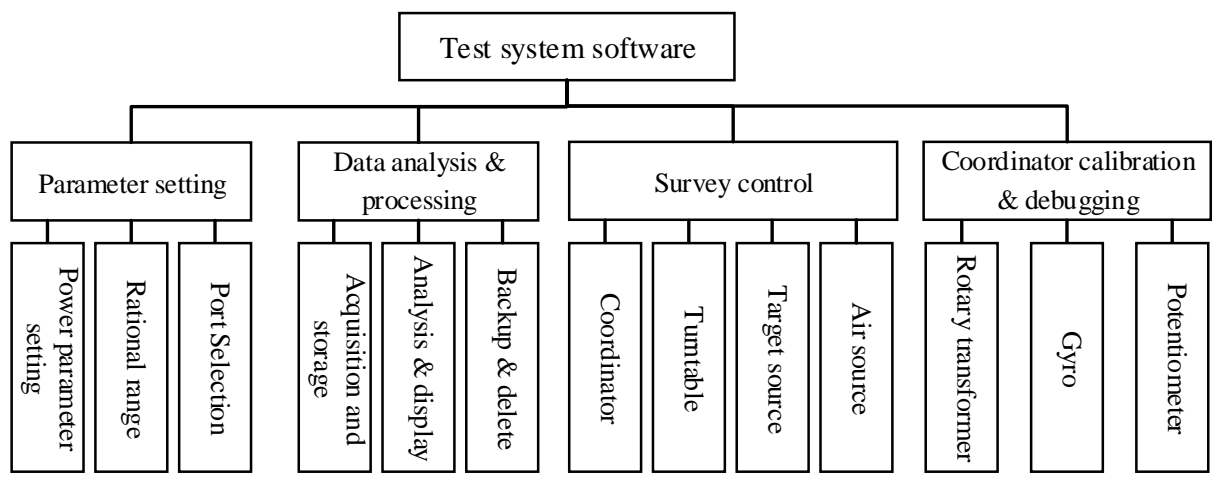

FIGURE II. SOFTWARE STRUCTURE OF TSPTS

System software consists of the following parts: parameter setting module, data analysis \& processing module, control module and calibration \& debugging module. On runtime of test system software, different components are powered on first to 
guarantee target seeker in normal operating conditions. Then, power parameter is set, turntable is controlled according to fixed motion pattern in standard working flow, and coordinator is controlled meanwhile for data analysis \& processing module to collect, display and record different parameter information of target seeker.

\section{Key Technologies In SOFTwARE DESIGN}

Software design takes the key part of the system. The involved technologies include multithreading, high-speed serial port communication, dynamic link library, etc.

\section{A. Multithreading Technology}

Multithreading is a technology used for parallel execution of multi-threads from software or hardware [9]. It could split up many missions and carry out synchronous implementation in multiple environments. Software of TSPTS uses multithreading technology to realize simultaneous processing of multi events as data acquisition, store, display, etc., just as shown in Figure III.

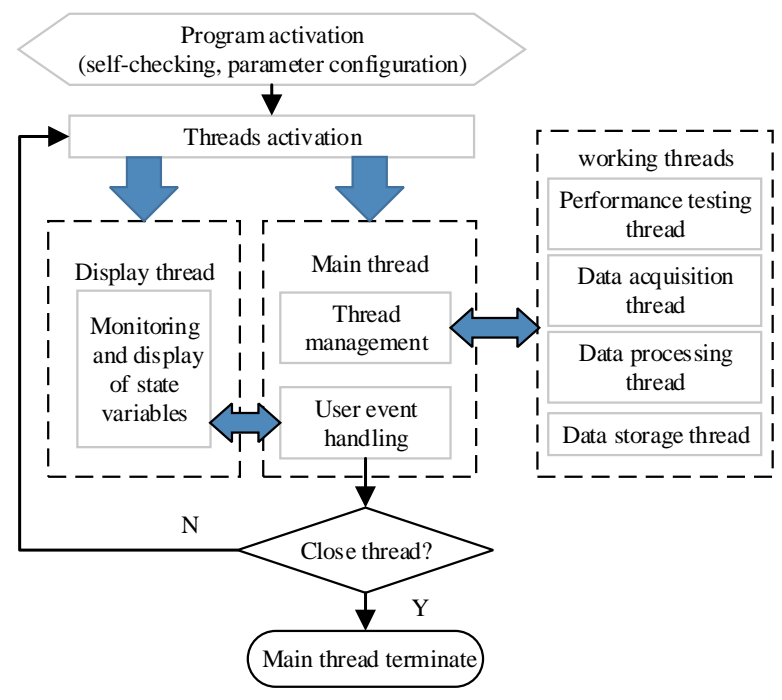

FIGURE III. WORKING-FLOW DIAGRAM OF THREADS

On program activation, self-checking of system and parameter configuration are performed first, after that display thread and main thread are launched. Display thread carries out monitoring and display of state variables acquired from coordinator, turntable, target source and air source. Main thread takes charge of user event handling and working condition control for different hardware. Besides, main thread creates the following 5 working threads. Performance testing thread accomplishes test of target seeker and invokes data acquisition thread. Data acquisition thread writes serial and LVDS data into memory sequentially, which also invokes data processing thread. Data processing thread reads data from acquisition memory and carries out processing analysis as required, which could also set flag bit of data storage thread to "1" at the same time. Data storage thread judges whether to store new data according to its flag bit and perform relevant operation.

\section{B. High Speed Serial Port Communication Technology}

Serial communication mode is adopted in target seeker test system, whose processing procedure mainly includes 4 steps as data framing, serial data transmit, data receiving and data analysis [10]. Data framing should be done first in communication process, where serial data frame structure is defined by communication protocol [11]. In software design, a "structure + union" mode is adopted to define data frame, which is transmitted to receiving-end in serial communication form.

On accepting transmitted data, receiving end carry out relevant processing immediately, which would accept new data after processing accomplished. By now, serial data frame transmission rate improves continually. When receiving and processing tasks of large size data frame remain in the same thread, data processing time could be inevitably long. So, data frames that fails to receive in time would be stored in buffer, and frame dropping would occur once buffer overflows. In allusion to frame dropping, software design of the system uses multithreading technology for cycling reading of serial port data frame, as shown in Figure IV.

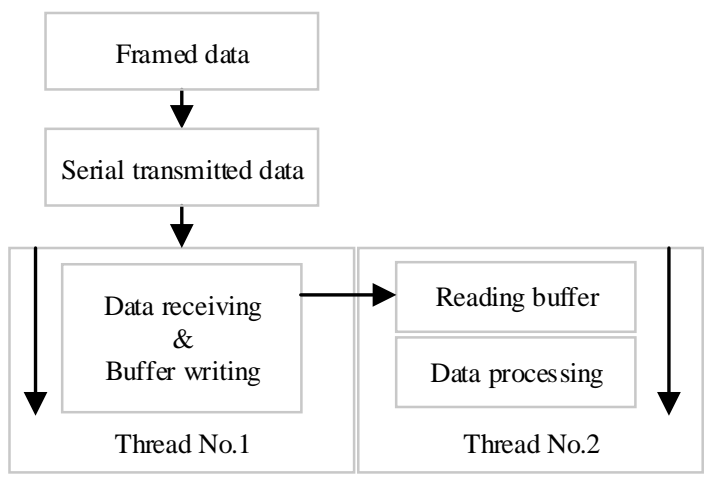

FIGURE IV. FLOW CHART OF SERIAL COMMUNICATION

Multithreading technology could assign time-consuming procedures of data receiving and handling to 2 different threads. One thread read data in input queue of serial port into receiving buffer. The other thread pick-up whole data frame from buffer according to protocol, which is then stored into thread-safe queue. While the residual data in buffer is less than a whole frame, it is saved for split-join with data in subsequent receiving cache to form a whole data frame.

\section{Dynamic Link Library Technology}

Dynamic link library is a kind of code sharing technology, which can be used to provide sharing function for other executable files. Application program using DLL can call derived functions of DLL, whereas, its executive code does not include performing code of these functions. TSPTS carries out communication with turntable computer through DLL file to control its moving.

Generally, DLL can be called with two modes as implicit link and explicit link [12]. Implicit link generates a corresponding lead-in LIB file automatically on building up a DLL file, which would be stored into EXE file. When functions 
of DLL are used, DLL is detected and loaded according to required information, which is then mapped to address space.

In contrast, explicit link does not designate which DLL is required on program compiling, but calls LoadLibrary function to load DLL after program execution. The address of output function is acquired by GetProcAddress after leading-in of DLL.

Implicit link implemented easily, whose link can be done at coding times, and derived functions of DLL can be called anywhere in the program. Still, with many implicit linked DLLs to load into memory at program activation, starting time would increase remarkably. Besides, a function of some DLL need to be called only under certain special condition at runtime of the program, so implicit link often lead to wasting of resources. Explicit link could determine which and when to load certain DLL file flexibly. Thus, time and memory cost on initial loading of application program can be reduced greatly. Therefore, explicit link is adopted in software design of TSPTS.

\section{SUMMARY}

The TSPTS designed in this paper takes full advantage of Virtual instrument technology. Functions as target seeker performance testing, turntable control, image acquisition \& display, calibration adjusting are realized using LabWindows/CVI development platform. The system integrates many tasks of measurement, control and monitoring together, which achieves a machine with multi-purpose function. The software of the system uses modular design, and each module takes on relevant function. Obviously, the system takes on good expansibility, whose maintenance and upgrade can be realized with unaltered hardware structure, which can greatly save human and material resources.

\section{REFERENCES}

[1] C. Wang, Y. Zhao, J.D. Zu, System design based on virtual instrument technology for measuring and testing vibration performance of seeker coordinator, Control \& Automation. 21 (2005) 159-160.

[2] Y. Li, Y. Zhao, Automatic Test System Based on Virtual Instrument Technology for Coordinator, Aero Weaponry. 1 (2008) 46-49.

[3] P. Zhang, Y. Zhao, Coordinator Performance Testing System based on LabVIEW, Metrology \& Measurement Technique. 38 (2011) 60-61,64.

[4] H.Y. Zheng, D.X. Dong, M.Y. Jia, Design of a Performance Test System for Infrared Steering Head Based on PXI Bus, Aero Weaponry. 4 (2010) 50-53.

[5] R. Chen, D.T. Yan, H. Ni, Design of gyro-rotor dynamical balance measurement system, Electronic Measurement Technology. 32 (2009) 179-183.

[6] Z.X. Xin, The Design of the Automatic Test System of Radar Seeker, Guidance \& Fuze. 26 (2005) 11-15.

[7] Information on http://www.ni.com.

[8] J.X. Wang, S.F. Yang, M.L. Sui, LabWindows/CVI Virtual Instrument Test Technology and Engineering Applications, 1st ed., Chemistry Industry Press, Beijing, 2011.

[9] NI corporation. Multithreading in LabWindows/CVI, Technical Publication, 2003.

[10] H.F. Lu, C.Y. Jiang, X.G. Yang, Key technical research of on-line monitor system based on serial communication, Chinese Journal of Scientific Instrument. 27 (2006) 2043-2044, 2053.

[11] B. Wang, L. Zhang, J. Deng, etc., An efficient data processing method based on high-speed serial communication, Techniques of Automation and Applications. 35 (2016) 57-60.
[12] L.G. Kong, D.W. Fan, R.B. Xia, Method on Ethernet communication between LabWindows/CVI with PLC via cross-platform DLL, Electric Drive. 46 (2016) 60-62, 66. 